nichts anderes bestimmt, in das Eigentum der Gesellschaft übergehen und in die im Entstehen begriffene medizinhistorische und naturwissenschaftsgeschichtliche Bibliothek derselben eingeordnet, deren Katalog periodisch zur Veröffentlichung gelangen wird. Diese Bibliothek steht den Mitgliedern unserer Gesellschaft nach einem noch aufzustellenden Reglement zur freien Benutzung zur Verfügung.

Einsendungen für die Bibliographie und Bibliothek sind an den Unterzeichneten (Pharmakologisches Institut der Universität, Gloriastraße 32, Zürich), mit bestem Dank, erbeten.

H. Fischer.

\title{
Zur Geschichte der Wissenschaften im Allgemeinen
}

Die Geschichte der Wissenschaften ist eine große Fuge, in der die Stimmen der Völker nach und nach zum Vorschein kommen.

Wenn man die Probleme des Aristoteles ansieht, so erstaunt man über die Gabe des Bemerkens und für was alles die Griechen Augen gehabt haben. Nur begehen sie den Fehler der Übereilung, da sie von dem Phänomen unmittelbar zur Erklärung schreiten, wodurch denn ganz unzulängliche theoretische Aussprüche zum Vorschein kommen. Dieses ist jedoch der allgemeine Fehler, der noch heutzutage begangen wird.

Um sich aus der grenzenlosen Vielfachheit, Zerstückelung und Verwicklung der modernen Naturlehre wieder ins Einfache zu retten, muß man sich immer die Frage vorlegen: wie würde sich Plato gegen die Natur, wie sie uns jetzt in ihrer größern Mannigfaltigkeit, bei aller gründlichen Einheit, erscheinen mag, benommen haben?

Man rühmt das achtzehnte Jahrhundert, daß es sich hauptsächlich mit Analyse abgegeben; dem neunzehnten bleibt nun die Aufgabe, die falschen obwaltenden Synthesen zu entdecken und deren Inhalt aufs neue zu analysieren.

Gœthe

Aus den Aphorismen zur Wissenschaftsgeschichte. 